\title{
Rivastigmine modestly improves dementia associated with Parkinson's disease, but has important adverse effects
}

Emre M, Aarsland D, Albanese A, et al. Rivastigmine for dementia associated with Parkinson's disease. New Engl J Med 2004;351:2509-18.

What is the effect of the dual cholinesterase inhibitor rivastigmine on dementia associated with Parkinson's disease?

METHODS

Design: Randomised controlled trial.

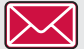

Allocation: Concealed.

Blinding: Double blinded.

$\sum<$

Follow up period: 24 weeks.

Setting: Centres in 11 European countries (including Turkey) and Canada; recruitment from October 2002 to July 2003

Patients: 541 people over the age of 50 years diagnosed with Parkinson's disease (PD; UK PD Society Brain Bank criteria) and mild to moderate PD related dementia (DSM-IV; Mini Mental State Examination (MMSE) score 10-24). Exclusion criteria were: non-PD neurodegeneration; unstable medical comorbidity, disability, or dementia not related to PD; active, uncontrolled seizures; prior major depressive disorder; or use of anticholinergic drugs or cholinesterase inhibitor in previous month.

$\mathbf{R}_{\mathbf{R}}$

Intervention: Rivastigmine (3-12 mg daily) titrated to maximum well tolerated dose over 16 week period, or placebo.

P(s)

Outcomes: Alzheimer's Disease Assessment Scale-cognitive subscale (ADAS-cog) score (range 0-70, higher score indicates greater impairment); Alzheimer's Disease Cooperative StudyClinician's Global Impression of Change (ADCS-CGIC; ranges from 1 (marked improvement) to 7 (marked worsening)); adverse events.

$\square$

Patient follow up: $75.8 \%$

\section{MAIN RESULTS}

Rivastigmine significantly improved cognitive performance compared with placebo by week 24 (mean change in ADAS-cog score: -2.1 with rivastigmine $v+0.7$ with placebo; $\mathrm{p}<0.001$ ). Rivastigmine significantly increased clinician rated global improvement by week 24 compared with placebo (mean ADCS-CGIC score: 3.8 with rivastigmine $v 4.3$ with placebo; $\mathrm{p}=0.007)$. However, rivastigmine was associated with significantly more nausea (29\% v $11.2 \%$; $\mathrm{p}<0.001)$, vomiting ( $16.6 \% \vee 1.7 \% ; \mathrm{p}<0.001)$, and tremor $(10.2 \% v$ $3.9 \%, \mathrm{p}=0.01$ ) than placebo. Serious adverse events were similar in both groups $(13.0 \%$ with rivastigmine $v 14.5 \%$ with placebo, $\mathrm{p}=0.69$ )

For correspondence: Dr Murat Emre, Istanbul Tip Fakültesi, Nöroloji Anabilim Dali, 34390 Capa, Istanbul, Turkey

Sources of funding: Novartis.

\section{CONCLUSIONS}

Rivastigmine produces modest but significant improvements in dementia associated with PD. Rivastigmine use is associated with an increase in nausea, vomiting, and tremor.

Commentary

ince the approval of cholinesterase inhibitors (ChE-Is) for sympto-

matic treatment of Alzheimer's disease (AD), both clinicians and pharmaceutical companies have been keen to understand whether people with other types of dementia or cognitive impairment will also benefit. There has been enthusiasm for administration of ChE-Is to people with dementia with Lewy bodies. ${ }^{1-3}$ Previous studies on Parkinson's disease (PD) had much smaller samples of at most 14 participants, and results raised questions about whether treatment with a cholinergic drug would secondarily worsen dopaminergic tone in those already at a primary dopaminergic disadvantage. ${ }^{4-}$

Although pharma-driven, the study design and methods used by Emre et al are fairly transparent. The study population was allowed to continue medication management as indicated for their parkinsonism and psychosis, and therefore represents a naturalistic sample of people with dementia in PD (PDD). One in 19 people with PDD who received rivastigmine had a clinically significant improvement on global measures of function (NNT = 19; compared with NNT of AD patients $=12$ )..$^{8}$ One in six people with PDD who received rivastigmine experienced nausea $(v$ one in 12 people with AD). ${ }^{8}$ More people with PDD developed worsening tremors than responded positively - the absence of post-treatment Unified Parkinson's Disease Rating Scale (UPDRS) scores for tremor is curious.

When contemplating the use of rivastigmine for PDD, the frequency of nausea should be borne in mind. In addition, the clinician should set realistic expectations by specifying target symptoms to evaluate during medication trial and noting that people with $A D$ have a higher response rate. The use of rivastigmine in people with tremor dominant PD or a Hoehn and Yahr scale score $>3$ would not be recommended, as this group was much less tolerant of the most frequent adverse events.

Emre et al have made an important contribution to the literature and seem to confirm concerns that some aspects of cholinergic treatment may not be beneficial to patients with PDD.

Tiffany Chow, MD

Clinician-Scientist, Rotman Research Institute; Assistant Professor of Neurology, Baycrest Centre for Geriatric Care, University of Toronto,

Canada

1 Lanctôt KL, Herrmann N. Donepezil for behavioural disorders associated with Lewy bodies: a case series. Int I Geriatr Psychiatry 2000;15:338-45.

2 McKeith I, Del Ser T, Spano P, et al. Efficacy of rivastigmine in dementia with Lewy bodies: a randomised, double-blind, placebo-controlled international study. Lancet 2000;356:2031-6.

3 Shea C, MacKnight C, Rockwood K. Donepezil for treatment of dementic with Lewy bodies: a case series of nine patients. Int Psychogeriatr 1998; 10:229-38

4 Mori S. Responses to donepezil in Alzheimer's disease and Parkinson's disease. Ann N Y Acad Sci 2002:977:493-500

5 Kurita A, Ochiai Y, Kono Y, et al. The beneficial effect of donepezil on visual hallucinations in three patients with Parkinson's disease. J Geriatr Psychiatry Neurol 2003;16:184-8

6 Fabbrini G, Barbanti P, Aurilia C, et al. Donepezil in the treatment of hallucinations and delusions in Parkinson's disease. Neurol Sci 2002:23:41-3.

7 Aarsland D, Laake K, Larsen JP, et al. Donepezil for cognitive impairment in Parkinson's disease: a randomised controlled study. I Neurol Neurosurg Psychiatry 2002;72:708-12.

8 Lanctôt KL, Herrmann N, Yau KK, et al. Efficacy and safety of cholinesterase inhibitors in Alzheimer's disease: a meta-analysis. Can Med Assoc J 2003;169:557-64. 